\title{
Robust Quantized Control of Hybrid Stochastic Systems based on Discrete-time Observations of State and Mode
}

\author{
Gongfei Song $^{\dagger *}$ Xuerong Mao ${ }^{\ddagger}$ Tao $\mathrm{Li}^{\dagger}$
}

\begin{abstract}
In this paper, the problems of robust quantized feedback control are studied for hybrid stochastic systems based on discrete-time observations of state and mode. All of the existing results in this area design the quantized feedback control based on continuous observations of the state and mode for all time $t \geq 0$ (see [23-25]). This is the first paper where we propose to use the quantized feedback control based on discrete-time observations of the state and mode. The key reason for this is to reduce the burden of communication by using not only the quantization (i.e. in the direction of state axis), but also discrete-time observations of state and mode (i.e. in the direction of time axis). Thus, the designed quantized feedback controllers have to be based on the discrete-time observations of state and mode. Clearly, the new quantized feedback controllers are more realistic and cost less in practice. Two examples with computer simulations will be provided to illustrate the effectiveness of the proposed control method.
\end{abstract}

Keywords Quantized control · Stochastic systems · Markov chain · Brownian motion · Mean-square exponentially stability

\section{Introduction}

Recently, the hybrid systems driven by continuous-time Markov chains have been intensively studied due to the reason that many practical systems can be modeled as hybrid stochastic systems, such as electric power systems, manufacturing systems, financial systems, networked control systems. An area of particular importance in the study of hybrid stochastic systems is stability analysis arising from automatic control (see, for example, [1-6], and the references therein). Moreover, discrete-time Markovian jump systems with polytopic-type parameter uncertainty and discrete-time nonlinear stochastic systems with mixed time delays were investigated in [5] and [6], while the linear matrix inequalities (LMIs) methods were proposed to design feedback controllers. The $H_{\infty}$ filtering problem in almost sure sense for nonlinear hybrid stochastic systems was addressed in [8]. It is well known that stochastic variables frequently exist in networked control systems. Some research results on this topic have been reported in $[9,10]$. In $[9]$, the robust stabilization of delayed Markovian jump systems was studied, which was applied to consider the robust synchronization of multi-agent network.

The stabilization problem of continuous-time hybrid stochastic systems has already been discussed in, for example, [12-17]. The mean-square exponential stabilization of the hybrid systems by delay feedback

\footnotetext{
${ }^{*}$ Corresponding author. Email address: gfsong@nuist.edu.cn

${ }^{\dagger}$ CICAEET, School of Information and Control, Nanjing University of Information Science and Technology, Nanjing 210044, Jiangsu, P. R. China.

${ }^{\ddagger}$ Department of Mathematics and Statistics, University of Strathclyde, Glasgow G1 1XH, UK.
} 
control was proposed in [12]. The study of the mean-square exponential stabilization of hybrid stochastic systems by feedback controls based on discrete-time state observations was initiated in [13], where an upper bound on the duration $\tau$ between two consecutive state observations was also given. Later, [14] continued to consider the stabilization problem of continuous-time hybrid stochastic systems and developed a better bound on $\tau$ by making full use of their special features. In particular, [15] showed that the discretetime stochastic feedback control stabilized an unstable deterministic system. In [16], the method of the Lyapunov functionals was introduced to study the mean square stability and the almost sure stability. More recently, [17] took a further step to investigate the issue of feedback control based on discrete-time observations of both state and mode.

On the other hand, one of the most important research areas in control theory is quantized control. Quantization is a peculiar characteristic of control systems. Therefore, quantized feedback can been found in many engineering systems including mechanical systems and networked systems. In the past decades, a great number of results in this area have appeared in the literature (see, e.g., [18-20]). By utilizing the classical sector bound approach, a logarithmic quantizer has been presented in [18]. Compared with logarithmic quantizer, a uniform quantizer was derived in $[19,20]$. It is worth noting that the quantized control problem for stochastic systems has been an active topic, many interesting results have been reported in [21-27]. The problem of a communication channel connecting the sensor to the controller for linear stochastic systems was considered in [21]. The quantized $H_{\infty}$ control problem for nonlinear stochastic time-delay systems with logarithmic quantizer was addressed in [22]. The sliding mode observer of Markovian jump systems was designed by using quantized measurements in [23]. Based on a mode-dependent logarithmic quantizer, the problem of filter design for uncertain stochastic systems was represented in [24]. The $H_{\infty}$ filtering problem of Markovian jump singular systems and the problem of finite-time bounded control for a class of stochastic nonlinear systems were provided in $[25,26]$, where the frameworks were proposed based on quantized output signal. As a special class of industrial systems, the networked Markovian jump systems were studied in [27], in which a quantizer was constructed between the sensor and the controller.

I should be emphasised that the literature mentioned above are all concerned with the quantized stabilization problem of continuous-time hybrid stochastic systems by continuous-time feedback controls. To the best knowledge of the authors, there is so far no result on this stabilization problem by discretetime feedback control. However, to reduce the control cost, it is better to observe both state and mode at discrete times based on which the quantized feedback control could be designed. This is the motivation for our current research.

In this paper, we consider the problem of robust quantized feedback control for hybrid stochastic systems based on discrete-time observations of state and mode. In the underlying system, both normbounded uncertainties and nonlinearity are taken into account simultaneously. It is worth pointing out that the nonlinearity is assumed to satisfy the global Lipschitz condition and the maximum admissible Lipschitz constant through convex optimization is obtained. Our work is based on logarithmic quantized feedback. We study quantization on the controller to the actuator side and the sensor to the controller side. For the former, a quantized feedback controller based on discrete-time observations of state and mode is the structure control of the form $u(x([t / \tau] \tau), r([t / \tau] \tau))=E(r(t)) q_{r(t)}(K(r([t / \tau] \tau)) x([t / \tau] \tau))$, where $\tau>0$ is a constant, $[t / \tau]$ is the integer part of $t / \tau$ and $q_{r(t)}(\cdot)$ is a mode-dependent quantizer. In this case, $E(\cdot)$ is given while $K(\cdot)$ needs to be designed. As is well known, this case corresponds to output injection (see [12]). For the latter, a quantized discrete-time-state-mode feedback controller is the structure control of the form $u(x([t / \tau] \tau), r([t / \tau] \tau))=E(r([t / \tau] \tau)) q_{r(t)}(K(r(t)) x([t / \tau] \tau))$. In this case, we 
are here required to design $E(\cdot)$ as $K(\cdot)$ is given. In addition, it corresponds to the case of state feedback (see [12]). Based on the correspoinding controllers, we will able to show the controlled hybrid stochastic systems are mean-square exponentially stable.

Notation: The notation used throughout this paper is standard. Let $\left(\Omega, \mathscr{F},\left\{\mathscr{F}_{t}\right\}_{t \geq 0}, \mathbb{P}\right)$ be a complete probability space with a filtration $\left\{\mathscr{F}_{t}\right\}_{t \geq 0}$ satisfying the usual conditions (i.e. it is right continuous with $\mathscr{F}_{0}$ containing all $\mathbb{P}$-null sets). For a matrix or vector $A, A^{T}$ denotes its transpose. The notation $M \geq N$ $(M>N)$ with $M$ and $N$ being symmetric matrices, means that the matrix $M-N$ is positive semi-definite (positive definite). For any vector $x, x^{(h)}$ represents the $h$-th component of vector $x$ and $|x|$ denotes its Euclidean norm. $\|A\|=\max \{|A x|:|x|=1\}$ means the operator norm of a matrix $A$. $\operatorname{diag}\{\cdots\}$ and $\star$ stand for a block-diagonal matrix and symmetric blocks. For a symmetric matrix $Q, \lambda_{\min }(Q)$ and $\lambda_{\max }(Q)$ refer to the smallest and largest eigenvalues of $Q$, respectively. Finally, we use the symbol $\operatorname{Sym}\{A\}$ to represent $A+A^{T}$.

\section{Problem Formulation and Preliminaries}

Consider the following uncertain hybrid stochastic systems on $t \geq 0$ :

$$
\begin{aligned}
d x(t)= & {[(A(r(t))+\Delta A(t, r(t))) x(t)+u(x([t / \tau] \tau), r([t / \tau] \tau))+f(x(t), r(t))] d t } \\
& +\sum_{k=1}^{m}\left[B_{k}(r(t))+\Delta B_{k}(t, r(t))\right] x(t) d w_{k}(t),
\end{aligned}
$$

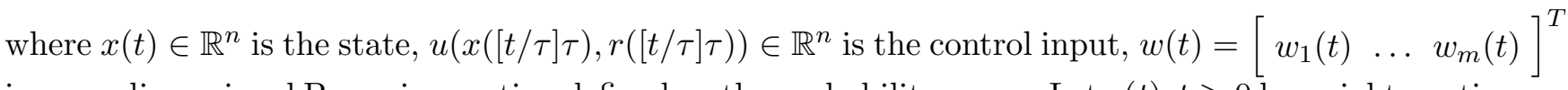
is an $m$-dimensional Brownian motion defined on the probability space. Let $r(t), t \geq 0$ be a right-continuous Markov chain on probability space taking values in a finite state space $\mathcal{S}=\{1,2, \ldots, N\}$ with generator $\Gamma=\left(\gamma_{i j}\right)_{N \times N}$ given by

$$
\mathbb{P}\{r(t+\tilde{\Delta})=j \mid r(t)=i\}= \begin{cases}\gamma_{i j} \tilde{\Delta}+o(\tilde{\Delta}), & \text { if } i \neq j, \\ 1+\gamma_{i i} \tilde{\Delta}+o(\tilde{\Delta}), & \text { if } i=j,\end{cases}
$$

where $\tilde{\Delta}>0$ and $\gamma_{i j} \geq 0$ is the transition rate from $i$ to $j$ if $i \neq j$, while $\gamma_{i i}=-\sum_{j \neq i} \gamma_{i j}$. We assume that the Markov chain $r(\cdot)$ is independent of the Brownian motion $w(\cdot)$. For any $i \in \mathcal{S}, k=1,2, \ldots, m, A(i) \triangleq A_{i}$ and $B_{k}(i) \triangleq B_{k i}$ are known real constant matrices with appropriate dimensions. $\Delta A(t, i) \triangleq \Delta A_{i}(t)$ and $\Delta B_{k}(t, i) \triangleq \Delta B_{k i}(t)$ are unknown matrices representing the structure of uncertainties, and are assumed to have the following properties

$$
\Delta A(t, i)=L_{a} F_{a}(t) N_{A i}, \quad \Delta B_{k}(t, i)=L_{b} F_{b}(t) N_{B k i}
$$

where $L_{a}, L_{b}, N_{A i}, N_{B k i}$ are known real constant matrices and $F_{a}(t), F_{b}(t): \mathbb{R}_{+} \rightarrow \mathbb{R}^{s \times t}$ are unknown real-valued time-varying matrices satisfying

$$
F_{a}(t)^{T} F_{a}(t) \leq I, \quad F_{b}(t)^{T} F_{b}(t) \leq I .
$$

Here $f(x(t), r(t)) \triangleq f_{i}(x(t)): \mathbb{R}^{n} \times \mathcal{S} \rightarrow \mathbb{R}^{n}$ is nonlinear function and assumed to be differentiable. As shown in [7], we make the following assumption on this nonlinear function. 
Assumption 1 We assume that the function $f_{i}(x(t))$ is globally Lipschitz with respect to $x(t)$ if $\left|f_{i}(0)\right|=0$ and

$$
\left|f_{i}\left(x_{1}(t)\right)-f_{i}\left(x_{2}(t)\right)\right| \leq \zeta\left|x_{1}(t)-x_{2}(t)\right|
$$

where $\zeta>0$ is called the Lipschitz constant.

Remark 1 Throughout this paper, it should be pointed out that the Lipschitz constant $\zeta>0$ is not fixed. The maximum allowable Lipschitz constant $\zeta^{*}$ can be determined by solving the convex optimization problem.

In this paper, a mode-dependent logarithmic quantizer under consideration is in the following form:

$$
\mathrm{q}_{i}(\nu)=\left[\begin{array}{llll}
\mathrm{q}_{i}^{(1)}\left(\nu^{(1)}\right) & \mathrm{q}_{i}^{(2)}\left(\nu^{(2)}\right) & \ldots & \mathrm{q}_{i}^{(l)}\left(\nu^{(l)}\right)
\end{array}\right]^{T}, \quad i \in \mathcal{S} .
$$

For each $q_{i}^{(r)}\left(\nu^{(r)}\right)(1 \leq r \leq l)$, the associated set of quantization levels is expressed as

$$
\mathcal{Q}^{r}=\left\{ \pm \mathcal{L}_{i}^{(r, j)} \mid \mathcal{L}_{i}^{(r, j)}=\left(\rho_{i}^{r}\right)^{j} \mathcal{L}_{i}^{(r, 0)}, j= \pm 1, \pm 2, \pm 3, \ldots\right\} \cup\left\{ \pm \mathcal{L}_{i}^{(r, 0)}\right\} \cup\{0\}, 0<\rho_{i}^{r}<1, \mathcal{L}_{i}^{(r, 0)}>0,
$$

where $\mathcal{L}_{i}^{(r, 0)}$ is the initial quantization values for the $r$-th sub-quantizer $q_{i}^{(r)}\left(\nu^{(r)}\right)$ and $\rho_{i}^{r}$ is the quantizer density of the $r$-th sub-quantizer $q_{i}^{(r)}\left(\nu^{(r)}\right)$. In this study, a characterization of the quantizer is given by

$$
q_{i}^{(r)}\left(\nu^{(r)}\right)= \begin{cases}\mathcal{L}_{i}^{(r, j)}, & \text { if } \frac{1}{1+\delta_{i}^{r}} \mathcal{L}_{i}^{(r, j)}<\nu^{(r)} \leq \frac{1}{1-\delta_{i}^{r}} \mathcal{L}_{i}^{(r, j)}, \quad \nu^{(r)}>0, \quad j= \pm 1, \pm 2, \pm 3, \ldots, \\ 0, & \text { if } \nu^{(r)}=0, \\ -q_{i}^{(r)}\left(-\nu^{(r)}\right), & \text { if } \nu_{(r)}<0, \quad r=1,2,3, \ldots, l,\end{cases}
$$

where $\delta_{i}^{r}=\frac{1-\rho_{i}^{r}}{1+\rho_{i}^{r}}$. It follows from [18] that, a sector bound expression can be proposed as

$$
q_{i}(\nu)=\left(I_{l}+\nabla_{i}(t)\right) \nu
$$

where the uncertainty matrix $\nabla_{i}(t)=\operatorname{diag}\left\{\nabla_{i}^{1}(t), \nabla_{i}^{2}(t), \ldots, \nabla_{i}^{l}(t)\right\}$ satisfies $\nabla_{i}^{r}(t) \in\left[-\delta_{i}^{r}, \delta_{i}^{r}\right], \quad r=$ $1,2, \ldots, l$.

Here, we cite the following definition (see [2]) that will be used in this paper.

Definition 1 The controlled hybrid stochastic system (1) with initial conditions $x(0)=x_{0} \in L_{\mathscr{F}_{0}}^{2}\left(\mathbb{R}^{n}\right)$, $r(0)=r_{0} \in \mathcal{S}$ is said to be exponentially stable in mean square, if there is a positive constant $\xi>0$ such that the solution $x(t)$ satisfies

$$
\limsup _{t \rightarrow \infty} \frac{1}{t} \log \left(\mathbb{E}|x(t)|^{2}\right) \leq-\xi
$$

Now, we consider two different quantized feedback controllers based on discrete-time state and mode observations.

- Case 1 Output injection

$$
u(x(\phi(t)), r(\phi(t)))=E(r(t)) q_{r(t)}(K(r(\phi(t))) x(\phi(t))),
$$


where $\phi(t)=[t / \tau] \tau$ for $t \geq 0$ and $q_{r(t)}(\cdot)$ is the mode-dependent logarithmic quantizer described above. It is well known that the controller sends the control output back to the actuator through an information channel. However, the bandwidth of the information channel is limited. To reduce the communication burden of the information channel, the mode-dependent logarithmic quantizer is constructed between the controller and the actuator. Here, $E(\cdot)$ is given so our aim is to design $K(\cdot)$ such that controlled system (1) is exponentially stable in mean square.

- Case 2 State feedback

$$
u(x(\phi(t)), r(\phi(t)))=E(r(\phi(t))) q_{r(t)}(K(r(t)) x(\phi(t))),
$$

where $\phi(t)=[t / \tau] \tau$ for $t \geq 0$ and $q_{r(t)}(\cdot)$ is the mode-dependent logarithmic quantizer mentioned above. In this case, the value of the system output is sent to the controller through a limited information channel. It is therefore known the mode-dependent logarithmic quantizer is constructed between the sensor and the controller. Furthermore, $K(\cdot)$ is given and our purpose is focused on the design of $E(\cdot)$ so that controlled system (1) is exponentially stable in mean square.

\section{Main Results}

Before proceeding further, we give the following lemmas which will be used in the proof of our main results. As is well known, almost all sample paths of Markov chain $r(t)$ are step-functions with a finite number of simple jumps in any finite subinterval of $\mathbb{R}_{+}$. In particular, Lemma 2 estimates the probability of jumps.

Lemma 1 ([11]) Suppose $A, M, N, W$ and $F(t)$ be real matrices of appropriate dimensions such that $W>0$ and $F(t)^{T} F(t) \leq I$. Then, we have the following.

(1) For any scalar $\varepsilon>0$ and vectors $x, y \in \mathbb{R}^{n}$,

$$
2 x^{T} M F(t) N y \leq \varepsilon^{-1} x^{T} M M^{T} x+\varepsilon y^{T} N^{T} N y .
$$

(2) For any scalar $\varepsilon>0$ such that $W^{-1}-\varepsilon M M^{T}>0$,

$$
[A+M F(t) N]^{T} W[A+M F(t) N] \leq A^{T}\left(W^{-1}-\varepsilon M M^{T}\right)^{-1} A+\varepsilon^{-1} N^{T} N .
$$

Lemma 2 For any $t \geq 0, v>0$ and $i \in \mathcal{S}$, we have

$$
\mathbb{P}(r(s) \neq i \text { for some } s \in[t, t+v] \mid r(t)=i) \leq 1-e^{-\hat{\gamma} v},
$$

where $\hat{\gamma}=\max _{i \in \mathcal{S}}\left(-\gamma_{i i}\right)$.

Proof Given $r(t)=i$, define the stopping time

$$
\sigma_{i}=\inf \{s \geq t: r(s) \neq i\},
$$

where and throughout this article we set $\inf \emptyset=\infty$ (in which $\emptyset$ denotes the empty set as usual). Inspired in the work of [1], $\sigma_{i}-t$ has the exponential distribution with parameter $-\gamma_{i i}$. Therefore,

$$
\begin{aligned}
& \mathbb{P}(r(s) \neq i \text { for some } s \in[t, t+v] \mid r(t)=i) \\
= & \mathbb{P}\left(\sigma_{i}-t \leq v \mid r(t)=i\right) \\
= & \int_{0}^{v}-\gamma_{i i} e^{\gamma_{i i} s} d s \\
= & 1-e^{\gamma_{i i} v} \leq 1-e^{-\hat{\gamma} v},
\end{aligned}
$$


as desired. This completes the proof.

The controlled system (1) is in fact a special hybrid stochastic system with a bounded variable delay, and the coefficients satisfy the Lipschitz condition and the linear growth condition. According to the existenceuniqueness theorem on stochastic differential equations with Markovian switching (see [2]), there exists a unique solution $x(t)$ to under initial conditions $x(0)=x_{0} \in L_{\mathscr{F}_{0}}^{2}\left(\mathbb{R}^{n}\right), r(0)=r_{0} \in \mathcal{S}$. Moreover, the solution satisfies $\mathbb{E}|x(t)|^{2}<\infty$ for $t \geq 0$. The following lemma will play important roles for the proof of our main results here.

Lemma 3 Let $x(t)$ be the solution of system (1). Denote

$$
\begin{aligned}
M_{a} & =2 \max _{i \in \mathcal{S}}\left(\left\|A_{i}\right\|^{2}+\left\|L_{a}\right\|^{2}\left\|N_{A i}\right\|^{2}\right), \quad M_{K}=2 \max _{i \in \mathcal{S}}\left(\left\|E_{i} K_{i}\right\|^{2}+\left\|E_{i} \Lambda_{i}\right\|^{2}\left\|K_{i}\right\|^{2}\right), \\
\Lambda_{i} & =\operatorname{diag}\left\{\delta_{i}^{1}, \delta_{i}^{2}, \ldots, \delta_{i}^{l},\right\}, \quad M_{b}=2 \max _{i \in \mathcal{S}} \sum_{k=1}^{m}\left(\left\|B_{k i}\right\|^{2}+\left\|L_{b}\right\|^{2}\left\|N_{B k i}\right\|^{2}\right),
\end{aligned}
$$

and define

$$
H(\tau)=\left(8 \tau^{2} M_{a}+8 \tau^{2} \zeta^{2}+8 \tau M_{b}+4 \tau^{2} M_{K}\right) e^{8 \tau^{2} M_{a}+8 \tau^{2} \zeta^{2}+8 \tau M_{b}}
$$

for $\tau>0$. If $\tau$ is small enough for $H(\tau)<\frac{1}{2}$, then we can obtain

$$
\mathbb{E}|x(t)-x(\phi(t))|^{2} \leq \frac{2 H(\tau)}{1-2 H(\tau)} \mathbb{E}|x(t)|^{2},
$$

for all $t \geq 0$.

Proof Fix an integer $\kappa \geq 0$. For any $t \in[\kappa \tau,(\kappa+1) \tau)$, applying the controller (8) to system (1), it can be seen that $\phi(t)=\kappa \tau$ and

$$
\begin{aligned}
x(t)-x(\phi(t))= & x(t)-x(\kappa \tau) \\
= & \int_{\kappa \tau}^{t}\left[(A(r(s))+\Delta A(s, r(s))) x(s)+E(r(s)) q_{r(s)}(K(r(\kappa \tau)) x(\kappa \tau))+f(x(s), r(s))\right] d s \\
& +\sum_{k=1}^{m} \int_{\kappa \tau}^{t}\left[B_{k}(r(s))+\Delta B_{k}(s, r(s))\right] x(s) d w_{k}(s) .
\end{aligned}
$$

Then, for any $r(s)=i \in \mathcal{S}$, it can be shown that

$$
\begin{aligned}
\mathbb{E}|x(t)-x(\phi(t))|^{2} \leq & 4 \mathbb{E}\left|\int_{\kappa \tau}^{t}\left(A_{i}+\Delta A_{i}(s)\right) x(s) d s\right|^{2}+4 \mathbb{E}\left|\int_{\kappa \tau}^{t} E_{i}\left(I+\nabla_{i}(s)\right) K(r(\phi(s))) x(\phi(s)) d s\right|^{2} \\
& +4 \mathbb{E}\left|\int_{\kappa \tau}^{t} f_{i}(x(s)) d s\right|^{2}+4 \mathbb{E}\left|\sum_{k=1}^{m} \int_{\kappa \tau}^{t}\left(B_{k i}+\Delta B_{k i}(s)\right) x(s) d w_{k}(s)\right|^{2}
\end{aligned}
$$

By using Assumption 1, Hölder inequality and Doob martingale inequality, we can derive the following four cases:

$$
\begin{aligned}
\text { (a) } \quad 4 \mathbb{E}\left|\int_{\kappa \tau}^{t}\left(A_{i}+\Delta A_{i}(s)\right) x(s) d s\right|^{2} & \leq 4 \tau \int_{\kappa \tau}^{t} \mathbb{E}\left(\left\|A_{i}+\Delta A_{i}(s)\right\|^{2}|x(s)|^{2}\right) d s \\
& \leq 4 \tau \int_{\kappa \tau}^{t} \mathbb{E}\left(\left(2|| A_{i}\left\|^{2}+2|| L_{a} F_{a}(s) N_{A i}\right\|^{2}\right)|x(s)|^{2}\right) d s \\
& \leq 4 \tau \int_{\kappa \tau}^{t} 2\left(\left\|A_{i}\right\|^{2}+|| L_{a}\left\|^{2}\right\| N_{A i} \|^{2}\right) \mathbb{E}|x(s)|^{2} d s \\
& \leq 4 \tau M_{a} \int_{\kappa \tau}^{t} \mathbb{E}|x(s)|^{2} d s
\end{aligned}
$$


(b)

$$
\begin{aligned}
& 4 \mathbb{E}\left|\int_{\kappa \tau}^{t} E_{i}\left(I+\nabla_{i}(s)\right) K(r(\phi(s))) x(\phi(s)) d s\right|^{2} \\
= & 4 \mathbb{E}\left|\int_{\kappa \tau}^{t}\left[E_{i} K(r(\phi(s))) x(\phi(s))+E_{i} \Lambda_{i} \Lambda_{i}^{-1} \nabla_{i}(s) K(r(\phi(s))) x(\phi(s))\right] d s\right|^{2} \\
\leq & 4 \tau \int_{\kappa \tau}^{t} 2\left(\left\|E_{i} K(r(\phi(s)))\right\|^{2}+\left\|E_{i} \Lambda_{i}\right\|^{2}|| K(r(\phi(s))) \|^{2}\right) \mathbb{E}|x(\phi(s))|^{2} d s \\
\leq & 4 \tau^{2} M_{K} \mathbb{E}|x(\kappa \tau)|^{2}
\end{aligned}
$$

(c) $\quad 4 \mathbb{E}\left|\int_{\kappa \tau}^{t} f_{i}(x(s)) d s\right|^{2} \leq 4 \tau \int_{\kappa \tau}^{t} \mathbb{E}\left|f_{i}(x(s))\right|^{2} d s \leq 4 \tau \zeta^{2} \int_{\kappa \tau}^{t} \mathbb{E}|x(s)|^{2} d s$,

$$
\begin{aligned}
\text { (d) } \quad 4 \mathbb{E}\left|\sum_{k=1}^{m} \int_{\kappa \tau}^{t}\left(B_{k i}+\Delta B_{k i}(s)\right) x(s) d w_{k}(s)\right|^{2} & \leq 4 \sum_{k=1}^{m} \int_{\kappa \tau}^{t} \mathbb{E}\left(\left\|B_{k i}+\Delta B_{k i}(s)\right\|^{2}|x(s)|^{2}\right) d s \\
& \leq 4 M_{b} \int_{\kappa \tau}^{t} \mathbb{E}|x(s)|^{2} d s .
\end{aligned}
$$

Thus, it is easy to see that (16) can be re-written as

$$
\begin{aligned}
\mathbb{E}|x(t)-x(\phi(t))|^{2} \leq & 4\left(\tau M_{a}+\tau \zeta^{2}+M_{b}\right) \int_{\kappa \tau}^{t} \mathbb{E}|x(s)|^{2} d s+4 \tau^{2} M_{K} \mathbb{E}|x(\phi(s))|^{2} \\
\leq & 8\left(\tau M_{a}+\tau \zeta^{2}+M_{b}\right) \int_{\kappa \tau}^{t} \mathbb{E}|x(s)-x(\phi(s))|^{2} d s \\
& +\left[8\left(\tau^{2} M_{a}+\tau^{2} \zeta^{2}+\tau M_{b}\right)+4 \tau^{2} M_{K}\right] \mathbb{E}|x(\kappa \tau)|^{2} .
\end{aligned}
$$

By applying Gronwall inequality, we can obtain

$$
\mathbb{E}|x(t)-x(\phi(t))|^{2} \leq H(\tau) \mathbb{E}|x(\kappa \tau)|^{2} .
$$

Therefore, it follows from (18) that

$$
\mathbb{E}|x(t)-x(\phi(t))|^{2} \leq 2 H(\tau)\left(\mathbb{E}|x(t)-x(\phi(t))|^{2}+\mathbb{E}|x(t)|^{2}\right),
$$

which implies (14) holds for $t \in[\kappa \tau,(\kappa+1) \tau)$. Then assertion (14) holds for all $t \geq 0$ as $\kappa \geq 0$ is arbitrary. This completes the proof.

Remark 2 Clearly, $H(\tau)$ is a continuous increasing function of $\tau$. It is easy to show that $H(0)=0$. Thus, if $\tau$ is small enough, then we can guarantee that $H(\tau)<\frac{1}{2}$.

Now, we are in a position to consider the case of output injection specified above.

Theorem 1 Assume that there exist matrices $K_{i}, Q_{i}>0$ and positive scalars $\beta_{i}, \varepsilon, \zeta, \beta_{2 i}, \eta_{i}(i \in \mathcal{S})$ such that

$$
\begin{aligned}
\bar{Q}_{i} \triangleq & \operatorname{Sym}\left\{Q_{i}\left(A_{i}+E_{i} K_{i}\right)\right\}+Q_{i} L_{a} \beta_{i}^{-1} L_{a}^{T} Q_{i}+N_{A i}^{T} \beta_{i} N_{A i}+Q_{i} \varepsilon^{-1} Q_{i}+\varepsilon \zeta^{2} I+Q_{i} E_{i} \Lambda_{i} \beta_{2 i}^{-1} \Lambda_{i}^{T} E_{i}^{T} Q_{i} \\
& +K_{i}^{T} \beta_{2 i} K_{i}+\sum_{j=1}^{N} \gamma_{i j} Q_{j}+\sum_{k=1}^{m}\left[B_{k i}^{T}\left(Q_{i}^{-1}-\eta_{i} L_{b} L_{b}^{T}\right)^{-1} B_{k i}+N_{B k i}^{T} \eta_{i}^{-1} N_{B k i}\right]
\end{aligned}
$$

are all negative-definite matrices, and $Q_{i}^{-1}-\eta_{i} L_{b} L_{b}^{T}>0$. Let $H(\tau)$ be the same as defined in Lemma 3 , and set

$$
\begin{aligned}
\bar{\lambda} & =\max _{i \in \mathcal{S}} \lambda_{\max }\left(\bar{Q}_{i}\right), \quad G_{Q E K}=\max _{i \in \mathcal{S}}\left\|Q_{i} E_{i} K_{i}\right\|, \quad \bar{G}_{Q E K}=\max _{i \in \mathcal{S}}\left\|Q_{i} E_{i} \Lambda_{i}\right\| \times\left\|K_{i}\right\|, \quad G_{E}=\max _{i \in \mathcal{S}}\left\|E_{i}\right\|, \\
\lambda_{m} & =\min _{i \in \mathcal{S}} \lambda_{\min }\left(Q_{i}\right), \quad \lambda_{M}=\max _{i \in \mathcal{S}} \lambda_{\max }\left(Q_{i}\right), \quad G_{K}=\max _{i, j \in \mathcal{S}, i \neq j}\left\|K_{i}-K_{j}\right\|^{2}, \quad G_{E \Lambda}=\max _{i \in \mathcal{S}}\left\|E_{i} \Lambda_{i}\right\| .
\end{aligned}
$$


If $\tau$ is sufficiently small for

$$
\chi(\tau) \triangleq \bar{\lambda}+2\left(G_{Q E K}+\bar{G}_{Q E K}\right) \sqrt{\frac{2 H(\tau)}{1-2 H(\tau)}}+2 \lambda_{M}\left(G_{E}+G_{E \Lambda}\right) \sqrt{\frac{2 G_{K}\left(1-e^{-\hat{\gamma} \tau}\right)}{1-2 H(\tau)}}<0,
$$

then the trajectories of system (1) satisfy

$$
\limsup _{t \rightarrow \infty} \frac{1}{t} \log \left(\mathbb{E}|x(t)|^{2}\right) \leq \frac{\chi(\tau)}{\lambda_{M}} .
$$

That is, the controlled system (1) is mean-square exponentially stable.

Proof We choose the Lyapunov function $V(x(t), r(t))=x(t)^{T} Q(r(t)) x(t)$ for system (1), where $Q(i) \triangleq Q_{i}$ as $r(t)=i$. Applying the generalized Itô formula to $V(x(t), r(t))$, we get

$$
d V(x(t), r(t))=\mathcal{L} V(x(t), r(t)) d t+d M_{1}(t)
$$

where $M_{1}(t)$ is a martingale with $M_{1}(0)=0$ and

$$
\begin{aligned}
\mathcal{L} V(x(t), i)= & 2 x(t)^{T} Q_{i}\left[\left(A_{i}+\Delta A_{i}(t)\right) x(t)+E_{i} q_{i}(K(r(\phi(t))) x(\phi(t)))+f_{i}(x(t))\right] \\
& +\sum_{k=1}^{m} x(t)^{T}\left(B_{k i}+\Delta B_{k i}(t)\right)^{T} Q_{i}\left(B_{k i}+\Delta B_{k i}(t)\right) x(t)+\sum_{j=1}^{N} \gamma_{i j} x(t)^{T} Q_{j} x(t) .
\end{aligned}
$$

By Lemma 1 and Assumption 1, we have

$$
\begin{aligned}
& 2 x(t)^{T} Q_{i}\left[\left(A_{i}+\Delta A_{i}(t)\right) x(t)+E_{i} q_{i}(K(r(\phi(t))) x(\phi(t)))+f_{i}(x(t))\right] \\
\leq & 2 x(t)^{T} Q_{i}\left(A_{i}+L_{a} F_{a}(t) N_{A i}\right) x(t)+2 x(t)^{T} Q_{i} E_{i} K(r(\phi(t))) x(\phi(t)) \\
& +2 x(t)^{T} Q_{i} E_{i} \nabla_{i}(t) K(r(\phi(t))) x(\phi(t))+x(t)^{T} Q_{i} \varepsilon^{-1} Q_{i} x(t)+f_{i}(x(t))^{T} \varepsilon f_{i}(x(t)) \\
\leq & x(t)^{T}\left\{\operatorname{Sym}\left\{Q_{i} A_{i}\right\}+\beta_{i}^{-1} Q_{i} L_{a} L_{a}^{T} Q_{i}+\beta_{i} N_{A i}^{T} N_{A i}+Q_{i} \varepsilon^{-1} Q_{i}+\varepsilon \zeta^{2} I\right\} x(t) \\
& +2 x(t)^{T} Q_{i} E_{i} K(r(\phi(t))) x(\phi(t))+2 x(t)^{T} Q_{i} E_{i} \nabla_{i}(t) K(r(\phi(t))) x(\phi(t)) .
\end{aligned}
$$

By some calculations, it can be verified that

$$
\begin{aligned}
& 2 x(t)^{T} Q_{i} E_{i} K(r(\phi(t))) x(\phi(t)) \\
= & 2 x(t)^{T} Q_{i} E_{i} K_{i} x(t)-2 x(t)^{T} Q_{i} E_{i} K_{i}(x(t)-x(\phi(t)))-2 x(t)^{T} Q_{i} E_{i}\left(K_{i}-K(r(\phi(t)))\right) x(\phi(t)) \\
\leq & x(t)^{T}\left(\operatorname{Sym}\left\{Q_{i} E_{i} K_{i}\right\}\right) x(t)+2 G_{Q E K}|x(t)| \times|x(t)-x(\phi(t))| \\
& -2 x(t)^{T} Q_{i} E_{i}\left(K_{i}-K(r(\phi(t)))\right) x(\phi(t)) .
\end{aligned}
$$


Setting $\pi_{1}=\sqrt{\frac{2 G_{E}^{2} G_{K}\left(1-e^{-\hat{\gamma} \tau}\right)}{1-2 H(\tau)}}$, and applying Lemmas 2 and 3, we further derive that

$$
\begin{aligned}
& -2 \mathbb{E}\left[x(t)^{T} Q_{i} E_{i}\left(K_{i}-K(r(\phi(t)))\right) x(\phi(t))\right] \\
\leq & \pi_{1} \lambda_{M} \mathbb{E}|x(t)|^{2}+\frac{\lambda_{M}}{\pi_{1}} \mathbb{E}\left(\| E_{i}||^{2}|| K_{i}-\left.K(r(\phi(t)))\right|^{2}|x(\phi(t))|^{2}\right) \\
= & \pi_{1} \lambda_{M} \mathbb{E}|x(t)|^{2}+\frac{\lambda_{M}}{\pi_{1}}|| E_{i} \|^{2} \mathbb{E}\left[\mathbb{E}\left(|| K_{i}-K(r(\phi(t))) \|^{2}|x(\phi(t))|^{2} \mid \mathscr{F}_{\phi(t)}\right)\right] \\
\leq & \pi_{1} \lambda_{M} \mathbb{E}|x(t)|^{2}+\frac{\lambda_{M}}{\pi_{1}} G_{E}^{2} \mathbb{E}\left[|x(\phi(t))|^{2} G_{K} \mathbb{E}\left(\sum_{j \in \mathcal{S}} I_{\{r(\phi(t))=j\}} I_{\{r(t) \neq j\}} \mid \mathscr{F}_{\phi(t)}\right)\right] \\
= & \pi_{1} \lambda_{M} \mathbb{E}|x(t)|^{2}+\frac{\lambda_{M}}{\pi_{1}} G_{E}^{2} \mathbb{E}\left[|x(\phi(t))|^{2} G_{K} \sum_{j \in \mathcal{S}} I_{\{r(\phi(t))=j\}} \mathbb{P}(r(t) \neq j \mid r(\phi(t))=j)\right] \\
\leq & \pi_{1} \lambda_{M} \mathbb{E}|x(t)|^{2}+\frac{\lambda_{M}}{\pi_{1}} G_{E}^{2}\left(1-e^{-\hat{\gamma} \tau}\right) G_{K} \mathbb{E}|x(\phi(t))|^{2} \\
\leq & \pi_{1} \lambda_{M} \mathbb{E}|x(t)|^{2}+\frac{\lambda_{M}}{\pi_{1}} G_{E}^{2}\left(1-e^{-\hat{\gamma} \tau}\right) G_{K} \frac{2}{1-2 H(\tau)} \mathbb{E}|x(t)|^{2} \\
= & 2 \pi_{1} \lambda_{M} \mathbb{E}|x(t)|^{2} .
\end{aligned}
$$

On the other hand, we can deduce that

$$
\begin{aligned}
& 2 x(t)^{T} Q_{i} E_{i} \nabla_{i}(t) K(r(\phi(t))) x(\phi(t)) \\
= & 2 x(t)^{T} Q_{i} E_{i} \nabla_{i}(t) K_{i} x(t)-2 x(t)^{T} Q_{i} E_{i} \nabla_{i}(t) K_{i}(x(t)-x(\phi(t))) \\
& -2 x(t)^{T} Q_{i} E_{i} \nabla_{i}(t)\left(K_{i}-K(r(\phi(t)))\right) x(\phi(t)) \\
\leq & x(t)^{T}\left(\beta_{2 i}^{-1} Q_{i} E_{i} \Lambda_{i} \Lambda_{i}^{T} E_{i}^{T} Q_{i}+\beta_{2 i} K_{i}^{T} K_{i}\right) x(t)+2 \bar{G}_{Q E K}|x(t)| \times|x(t)-x(\phi(t))| \\
& -2 x(t)^{T} Q_{i} E_{i} \nabla_{i}(t)\left(K_{i}-K(r(\phi(t)))\right) x(\phi(t)) .
\end{aligned}
$$

Similar to the derivation of $(25)$, it is easy to show that for $\pi_{2}=\sqrt{\frac{2 G_{E \Lambda}^{2} G_{K}\left(1-e^{-\hat{\gamma} \tau}\right)}{1-2 H(\tau)}}$,

$$
\begin{aligned}
& -2 \mathbb{E}\left[x(t)^{T} Q_{i} E_{i} \nabla_{i}(t)\left(K_{i}-K(r(\phi(t)))\right) x(\phi(t))\right] \\
\leq & \pi_{2} \lambda_{M} \mathbb{E}|x(t)|^{2}+\frac{\lambda_{M}}{\pi_{2}} \mathbb{E}\left(\left\|E_{i} \Lambda_{i}\right\|^{2}|| K_{i}-K(r(\phi(t))) \|^{2}|x(\phi(t))|^{2}\right) \\
\leq & 2 \pi_{2} \lambda_{M} \mathbb{E}|x(t)|^{2} .
\end{aligned}
$$

By applying Lemma 1 again, it follows that

$$
\begin{aligned}
& \sum_{k=1}^{m} x(t)^{T}\left(B_{k i}+\Delta B_{k i}(t)\right)^{T} Q_{i}\left(B_{k i}+\Delta B_{k i}(t)\right) x(t) \\
\leq & \sum_{k=1}^{m} x(t)^{T}\left[B_{k i}^{T}\left(Q_{i}^{-1}-\eta_{i} L_{b} L_{b}^{T}\right)^{-1} B_{k i}+\eta_{i}^{-1} N_{B k i}^{T} N_{B k i}\right] x(t) .
\end{aligned}
$$

Applying the generalized Itô formula now to $e^{\alpha t} x(t)^{T} Q(r(t)) x(t)$, we can obtain

$$
d\left[e^{\alpha t} x(t)^{T} Q(r(t)) x(t)\right]=e^{\alpha t}\left(\alpha x(t)^{T} Q(r(t)) x(t)+\mathcal{L} V(x(t), r(t))\right) d t+d M_{2}(t),
$$

where $M_{2}(t)$ is a continuous martingale with $M_{2}(0)=0$ and $\alpha=-\frac{\chi(\tau)}{\lambda_{M}}$. Then, it follows from (22)-(28) 
and (29) that

$$
\begin{aligned}
e^{\alpha t} \mathbb{E}\left(x(t)^{T} Q(r(t)) x(t)\right) \leq & \lambda_{M} \mathbb{E}|x(0)|^{2}+\int_{0}^{t} e^{\alpha s}\left(\alpha \lambda_{M}+\bar{\lambda}+2 \lambda_{M} \pi_{1}+2 \lambda_{M} \pi_{2}\right) \mathbb{E}|x(s)|^{2} d s \\
& +\int_{0}^{t} e^{\alpha s}\left[2 G_{Q E K} \mathbb{E}(|x(s)| \times|x(s)-x(\phi(s))|)\right. \\
& \left.+2 \bar{G}_{Q E K} \mathbb{E}(|x(s)| \times|x(s)-x(\phi(s))|)\right] d s \\
\leq & \lambda_{M} \mathbb{E}|x(0)|^{2}+\int_{0}^{t} e^{\alpha s}\left(\alpha \lambda_{M}+\bar{\lambda}+2 \lambda_{M} \pi_{1}+2 \lambda_{M} \pi_{2}\right) \mathbb{E}|x(s)|^{2} d s \\
& +\int_{0}^{t} e^{\alpha s}\left[\pi_{3} \mathbb{E}|x(s)|^{2}+\frac{G_{Q E K}^{2}}{\pi_{3}} \mathbb{E}|x(s)-x(\phi(s))|^{2}\right. \\
& \left.+\pi_{4} \mathbb{E}|x(s)|^{2}+\frac{\bar{G}_{Q E K}^{2}}{\pi_{4}} \mathbb{E}|x(s)-x(\phi(s))|^{2}\right] d s \\
\leq & \lambda_{M} \mathbb{E}|x(0)|^{2}+\int_{0}^{t} e^{\alpha s}\left(\alpha \lambda_{M}+\bar{\lambda}+2 \lambda_{M} \pi_{1}+2 \lambda_{M} \pi_{2}\right. \\
& \left.+2 \pi_{3}+2 \pi_{4}\right) \mathbb{E}|x(s)|^{2} d s \\
= & \lambda_{M} \mathbb{E}|x(0)|^{2},
\end{aligned}
$$

where $\pi_{3}=\sqrt{\frac{2 G_{Q E K}^{2} H(\tau)}{1-2 H(\tau)}}$ and $\pi_{4}=\sqrt{\frac{2 \bar{G}_{Q E K}^{2} H(\tau)}{1-2 H(\tau)}}$. By $(30)$, it is easy to see that $\limsup _{t \rightarrow \infty} \frac{1}{t} \log \left(\mathbb{E}|x(t)|^{2}\right) \leq$ $-\alpha=\frac{\chi(\tau)}{\lambda_{M}}$ holds; therefore, it follows from Definition 1 that the controlled system (1) is exponentially stable in mean square. This completes the proof.

Remark 3 First, we note that $H(\tau)$ is a continuous increasing function of $\tau$. Consequently, we have that $\chi(\tau)$ is also a increasing function of $\tau$. If we let $\tau=0$, then $\chi(0)=\bar{\lambda}<0$ is clearly true. Therefore, we set $\tau^{*}$ be the largest positive scalar such that the equation $\chi(\tau) \leq 0$, Then, we can obtain that $\chi(\tau)<0$ with $\forall \tau \in\left(0, \tau^{*}\right)$.

Remark 4 In this case, our aim is focused on the design of $K_{i}$ such that for any $i \in \mathcal{S}$, the controlled system (1) is exponentially stable in mean square. Moreover, we can transfer requirements (20) into LMIs. Firstly, pre- and post-multiplying $\bar{Q}_{i}$ by $Q_{i}^{-1}$, respectively, and then applying the Schur complement equivalence, we can obtain

$$
\left[\begin{array}{cccccccc}
\mathcal{A}_{i} & \mathcal{B}_{i} & \mathcal{N}_{i} & \mathcal{T}_{i} & X_{i} & I & X_{i} N_{A i}^{T} & \mathcal{K}_{i}^{T} \\
\star & \mathcal{Z}_{i} & 0 & 0 & 0 & 0 & 0 & 0 \\
\star & \star & \mathcal{J}_{i} & 0 & 0 & 0 & 0 & 0 \\
\star & \star & \star & \mathcal{X}_{i} & 0 & 0 & 0 & 0 \\
\star & \star & \star & \star & -\theta I & 0 & 0 & 0 \\
\star & \star & \star & \star & \star & -\varepsilon I & 0 & 0 \\
\star & \star & \star & \star & \star & \star & -\beta_{i}^{-1} I & 0 \\
\star & \star & \star & \star & \star & \star & \star & -\beta_{2 i}^{-1} I
\end{array}\right]<0
$$

where

$$
\begin{aligned}
& \mathcal{A}_{i}=\operatorname{Sym}\left\{A_{i} X_{i}+E_{i} \mathcal{K}_{i}\right\}+L_{a} \beta_{i}^{-1} L_{a}^{T}+E_{i} \Lambda_{i} \beta_{2 i}^{-1} \Lambda_{i}^{T} E_{i}^{T}+\gamma_{i i} X_{i}, \quad \mathcal{B}_{i}=\left[\begin{array}{lll}
X_{i} B_{1 i}^{T} & \cdots & X_{i} B_{m i}^{T}
\end{array}\right], \\
& \mathcal{Z}_{i}=\operatorname{diag}\left\{\eta_{i} L_{b} L_{b}^{T}-X_{i}, \ldots, \eta_{i} L_{b} L_{b}^{T}-X_{i}\right\}, \quad \mathcal{N}_{i}=\left[\begin{array}{lll}
X_{i} N_{B 1 i}^{T} & \cdots & X_{i} N_{B m i}^{T}
\end{array}\right],
\end{aligned}
$$

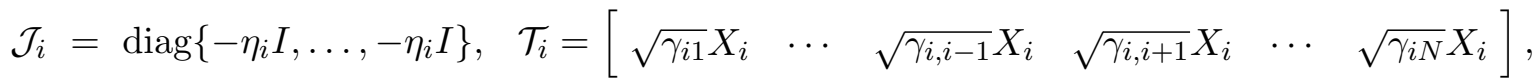

$$
\begin{aligned}
& \mathcal{X}_{i}=\operatorname{diag}\left\{-X_{1}, \ldots,-X_{i-1},-X_{i+1}, \ldots,-X_{N}\right\}, \quad \mathcal{K}_{i}=K_{i} X_{i}, \quad X_{i}=Q_{i}^{-1}, \quad \theta=\left(\varepsilon \zeta^{2}\right)^{-1}, \quad i \in \mathcal{S} .
\end{aligned}
$$


Remark 5 To optimize the corresponding Lipschitz constant, we can solve the following optimization problem

$$
\inf _{K_{i}, Q_{i}, \beta_{i}, \beta_{2 i}, \varepsilon, \theta, \eta_{i}, i \in \mathcal{S}} \varpi \theta+(1-\varpi) \varepsilon
$$

s. t. Inequalities (31),

where $\varpi$ is a tuning parameter with $0 \leq \varpi \leq 1$. Then, the maximum allowable Lipschitz constant is $\zeta^{*}=\frac{1}{\sqrt{\theta \varepsilon}}$.

We obtain the sufficient condition for the case of state feedback in the following theorem.

Theorem 2 Assume that there exist matrices $E_{i}, Q_{i}>0$ and positive scalars $\beta_{i}, \varepsilon, \zeta, \beta_{2 i}, \eta_{i}(i \in \mathcal{S})$ such that $\bar{Q}_{i}$ are all negative-definite matrices. Let $H(\tau), \bar{\lambda}, G_{Q E K}, \bar{G}_{Q E K}, \lambda_{m}$ and $\lambda_{M}$ be the same as defined in Theorem 1, and set $\tilde{G}_{E}=\max _{i, j \in \mathcal{S}, i \neq j}\left\|E_{i}-E_{j}\right\|^{2}, \tilde{G}_{K}=\max _{i \in \mathcal{S}}\left\|K_{i}\right\|, G_{\Lambda K}=\max _{i \in \mathcal{S}}\left\|\Lambda_{i} K_{i}\right\|$. If $\tau$ is sufficiently small for

$$
\varkappa(\tau) \triangleq \bar{\lambda}+2\left(G_{Q E K}+\bar{G}_{Q E K}\right) \sqrt{\frac{2 H(\tau)}{1-2 H(\tau)}}+2 \lambda_{M}\left(\tilde{G}_{K}+G_{\Lambda K}\right) \sqrt{\frac{2 \tilde{G}_{E}\left(1-e^{-\hat{\gamma} \tau}\right)}{1-2 H(\tau)}}<0,
$$

then the trajectories of system (1) satisfy

$$
\limsup _{t \rightarrow \infty} \frac{1}{t} \log \left(\mathbb{E}|x(t)|^{2}\right) \leq \frac{\varkappa(\tau)}{\lambda_{M}} .
$$

In other words, the controlled system (1) is exponentially stable in mean square.

Proof Now, we apply controller (9) for system (1), and obtain inequality (14). Applying the generalized Itô formula to $x(t)^{T} Q(r(t)) x(t)$, we have

$$
\begin{aligned}
d\left(x(t)^{T} Q(r(t)) x(t)\right)= & \left\{2 x(t)^{T} Q_{i}\left[\left(A_{i}+\Delta A_{i}(t)\right) x(t)+E(r(\phi(t))) q_{i}\left(K_{i} x(\phi(t))\right)+f_{i}(x(t))\right]\right. \\
& +\sum_{k=1}^{m} x(t)^{T}\left(B_{k i}+\Delta B_{k i}(t)\right)^{T} Q_{i}\left(B_{k i}+\Delta B_{k i}(t)\right) x(t) \\
& \left.+\sum_{j=1}^{N} \gamma_{i j} x(t)^{T} Q_{j} x(t)\right\} d t+d M_{3}(t),
\end{aligned}
$$

where $M_{3}(t)$ is also a martingale with $M_{3}(0)=0$. Actually, it can be seen that

$$
\begin{aligned}
& 2 x(t)^{T} Q_{i}\left[\left(A_{i}+\Delta A_{i}(t)\right) x(t)+E(r(\phi(t))) q_{i}\left(K_{i} x(\phi(t))\right)+f_{i}(x(t))\right] \\
\leq & x(t)^{T}\left\{\operatorname{Sym}\left\{Q_{i} A_{i}\right\}+\beta_{i}^{-1} Q_{i} L_{a} L_{a}^{T} Q_{i}+\beta_{i} N_{A i}^{T} N_{A i}+Q_{i} \varepsilon^{-1} Q_{i}+\varepsilon \zeta^{2} I\right\} x(t) \\
& +2 x(t)^{T} Q_{i} E(r(\phi(t))) K_{i} x(\phi(t))+2 x(t)^{T} Q_{i} E(r(\phi(t))) \nabla_{i}(t) K_{i} x(\phi(t)) .
\end{aligned}
$$

Then, by using Lemmas (1)-(3), it can be deduced that

$$
\begin{aligned}
& \mathbb{E}\left[2 x(t)^{T} Q_{i} E(r(\phi(t))) K_{i} x(\phi(t))\right] \\
\leq & \mathbb{E}\left[x(t)^{T}\left(\operatorname{Sym}\left\{Q_{i} E_{i} K_{i}\right\}\right) x(t)\right]+\left(2 \tilde{\pi}_{1} \lambda_{M}+2 \pi_{3}\right) \mathbb{E}|x(t)|^{2}, \\
& \mathbb{E}\left[2 x(t)^{T} Q_{i} E(r(\phi(t))) \nabla_{i}(t) K_{i} x(\phi(t))\right] \\
\leq & \mathbb{E}\left[x(t)^{T}\left(\beta_{2 i}^{-1} Q_{i} E_{i} \Lambda_{i} \Lambda_{i}^{T} E_{i}^{T} Q_{i}+\beta_{2 i} K_{i}^{T} K_{i}\right) x(t)\right]+\left(2 \tilde{\pi}_{2} \lambda_{M}+2 \pi_{4}\right) \mathbb{E}|x(t)|^{2},
\end{aligned}
$$


with $\tilde{\pi}_{1}=\sqrt{\frac{2 \tilde{G}_{K}^{2} \tilde{G}_{E}\left(1-e^{-\hat{\gamma} \tau}\right)}{1-2 H(\tau)}}$ and $\tilde{\pi}_{2}=\sqrt{\frac{2 G_{\Lambda K}^{2} \tilde{G}_{E}\left(1-e^{-\hat{\gamma} \tau}\right)}{1-2 H(\tau)}}$. In addition, from (34)-(37) and following a similar line as in the proof of Theorem 1, it can be verified that $\limsup _{t \rightarrow \infty} \frac{1}{t} \log \left(\mathbb{E}|x(t)|^{2}\right) \leq \frac{\varkappa(\tau)}{\lambda_{M}}$ holds. This completes the proof.

Similar to the case of output injection, we hence introduce the following remark.

Remark 6 Here, our aim is to design $E_{i}$ such that for any $i \in \mathcal{S}$, the controlled system (1) is exponentially stable in mean square. Furthermore, we convert requirements (20) into LMIs. Applying the Schur complement equivalence, we can obtain

$$
\left[\begin{array}{ccccccc}
\Xi_{i} & Q_{i} L_{a} & Q_{i} & I & \mathcal{E}_{i} \Lambda_{i} & \Pi_{i} & 0 \\
\star & -\beta_{i} I & 0 & 0 & 0 & 0 & 0 \\
\star & \star & -\varepsilon I & 0 & 0 & 0 & 0 \\
\star & \star & \star & -\theta I & 0 & 0 & 0 \\
\star & \star & \star & \star & -\beta_{2 i} I & 0 & 0 \\
\star & \star & \star & \star & \star & \Upsilon_{i} & \Phi_{i} \\
\star & \star & \star & \star & \star & \star & \Psi_{i}
\end{array}\right]<0,
$$

where

$$
\begin{aligned}
& \Xi_{i}=\operatorname{Sym}\left\{Q_{i} A_{i}+\mathcal{E}_{i} K_{i}\right\}+N_{A i}^{T} \beta_{i} N_{A i}+K_{i}^{T} \beta_{2 i} K_{i}+\sum_{j=1}^{N} \gamma_{i j} Q_{j}+\sum_{k=1}^{m} N_{B k i}^{T} \eta_{i}^{-1} N_{B k i}, \\
& \Pi_{i}=\left[\begin{array}{ccc}
B_{1 i}^{T} Q_{i} & \cdots & B_{m i}^{T} Q_{i}
\end{array}\right], \quad \Upsilon_{i}=\operatorname{diag}\left\{-Q_{i}, \ldots,-Q_{i}\right\}, \\
& \Phi_{i}=\operatorname{diag}\left\{Q_{i} L_{b}, \ldots, Q_{i} L_{b}\right\}, \quad \Psi_{i}=\operatorname{diag}\left\{-\eta_{i}^{-1}, \ldots,-\eta_{i}^{-1}\right\}, \quad \mathcal{E}_{i}=Q_{i} E_{i}, \quad i \in \mathcal{S} .
\end{aligned}
$$

Similar to (32), we can get the maximum allowable Lipschitz constant $\zeta^{*}$ by solving the corresponding optimization problem. For brevity, it is not presented here.

\section{Examples}

In this section, we provide two examples with computer simulations to demonstrate the effectiveness of the proposed method.

Example 1. Consider a two-dimensional uncertain hybrid stochastic system (1) with parameters as follows:

$$
\begin{aligned}
A_{1} & =\left[\begin{array}{cc}
-1.4 & 0.1 \\
0.3 & 0.5
\end{array}\right], \quad A_{2}=\left[\begin{array}{cc}
-0.6 & 0.1 \\
-0.1 & 0.5
\end{array}\right], \quad B_{1}=\left[\begin{array}{cc}
0.3 & 0.9 \\
0 & 0.1
\end{array}\right], \quad B_{2}=\left[\begin{array}{ll}
0.1 & 0.3 \\
0.5 & 0.2
\end{array}\right], \quad L_{a}=\left[\begin{array}{l}
0.3 \\
0.2
\end{array}\right], \\
N_{A 1} & =\left[\begin{array}{ll}
0.2 & 0.1
\end{array}\right], \quad N_{A 2}=\left[\begin{array}{ll}
0.1 & 0.3
\end{array}\right], \quad L_{b}=\left[\begin{array}{c}
0.2 \\
0.5
\end{array}\right], \quad N_{B 11}=\left[\begin{array}{ll}
0.1 & 0.5
\end{array}\right], \\
N_{B 12} & =\left[\begin{array}{ll}
0.2 & 0.6
\end{array}\right], \quad F_{a}(t)=\sin (t), \quad F_{b}(t)=\cos (t), \quad E_{1}=\left[\begin{array}{l}
0.2 \\
0.1
\end{array}\right], \quad E_{2}=\left[\begin{array}{l}
0.1 \\
0.2
\end{array}\right] .
\end{aligned}
$$

In this case, we assume that $\rho_{1}^{1}=0.3, \rho_{2}^{1}=0.6, m=1$. Here, $w(t)$ is a scalar Brownian motion and $r(t)$ is a Markov chain on the state space $\mathcal{S}=\{1,2\}$ with the generator $\Gamma=\left[\begin{array}{cc}-1.5 & 1.5 \\ 1 & -1\end{array}\right]$. For initial conditions $r(0)=1, x(0)=\left[\begin{array}{c}-2 \\ 8\end{array}\right]$, the result in Figure 1 shows that the open-loop system (1) (that is 

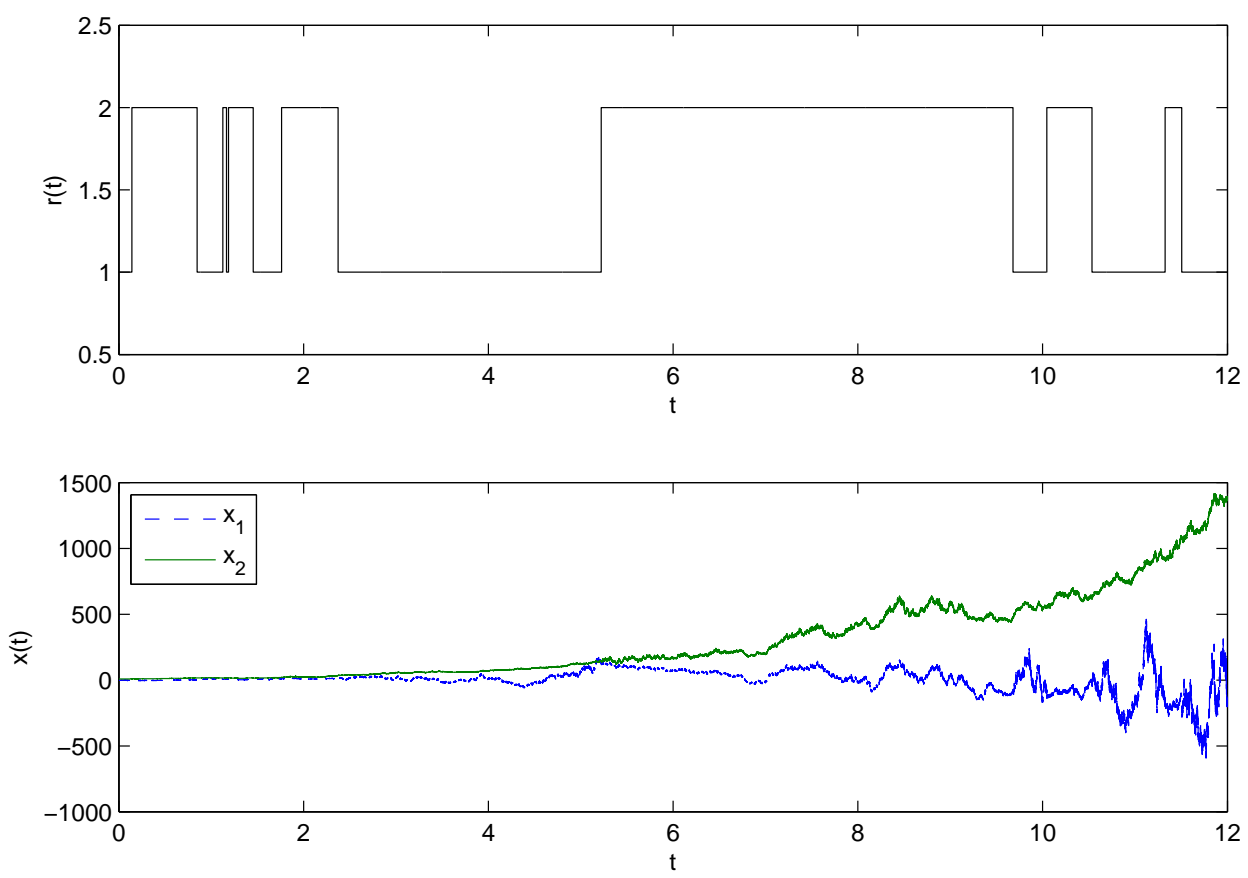

Figure 1: The trajectories of the open-loop system

$u(x([t / \tau] \tau), r([t / \tau] \tau))=0)$ is not mean-square exponentially stable. Our aim here is to seek for $K_{1}$ and $K_{2}$ in $\mathbb{R}^{1 \times 2}$ and then make sure $\tau$ is sufficiently small for the controlled system (1) to be exponentially stable in mean square. By solving the LMIs (31), we obtain a desired feedback controller in the form of (8) with

$$
K_{1}=\left[\begin{array}{ll}
-2.7070 & -13.7153
\end{array}\right], \quad K_{2}=\left[\begin{array}{ll}
-3.1602 & -16.2847
\end{array}\right] .
$$

Furthermore, it is easy to compute

$$
\begin{aligned}
\bar{\lambda} & =-0.01, \quad G_{Q E K}=0.2434, \quad \bar{G}_{Q E K}=0.1311, \quad M_{a}=4.1131, \quad \zeta=0.0051, \\
M_{b} & =1.9688, \quad M_{K}=29.2376, \quad \lambda_{M}=0.1421, \quad G_{E}=0.05, \quad G_{E \Lambda}=0.1204, \quad G_{K}=6.8070 .
\end{aligned}
$$

It is also easy to show that (21) holds whenever $\tau^{*}=0.0000046$. So by Theorem 1 , if we set $K_{1}, K_{2}$ as above and guarantee $\tau<\tau^{*}$, then the controlled system (1) is mean-square exponentially stable.

The mode-dependent quantizer parameters $\mathcal{L}_{1}^{(1,0)}, \mathcal{L}_{2}^{(1,0)}$ are selected as $\mathcal{L}_{1}^{(1,0)}=\mathcal{L}_{2}^{(1,0)}=30$. The nonlinear functions are assumed to be $f_{1}(x(t))=f_{2}(x(t))=\left[\begin{array}{c}0.005 \sin \left(e^{-x^{(2)}(t)}\right)+0.0051 \cos \left(x^{(1)}(t)\right) \\ 0.0051 \sin \left(e^{-x^{(2)}(t)}\right)\end{array}\right]$. The simulation results of trajectories of the closed-loop system (1) are recorded in Figure 2, from which we clearly see that the trajectories of the closed-loop system converge to the origin for initial values $r(0)=1$, $x(0)=\left[\begin{array}{c}-2 \\ 8\end{array}\right]$. In addition, solving the optimization problem (32), we can obtain the maximum allowable Lipschitz constant $\zeta^{*}=0.7383$.

Example 2. Consider an uncertain hybrid stochastic system (1) with the same system parameters as in Example 1 and

$$
K_{1}=\left[\begin{array}{ll}
0.2 & 1
\end{array}\right], \quad K_{2}=\left[\begin{array}{ll}
0 & 1
\end{array}\right] .
$$



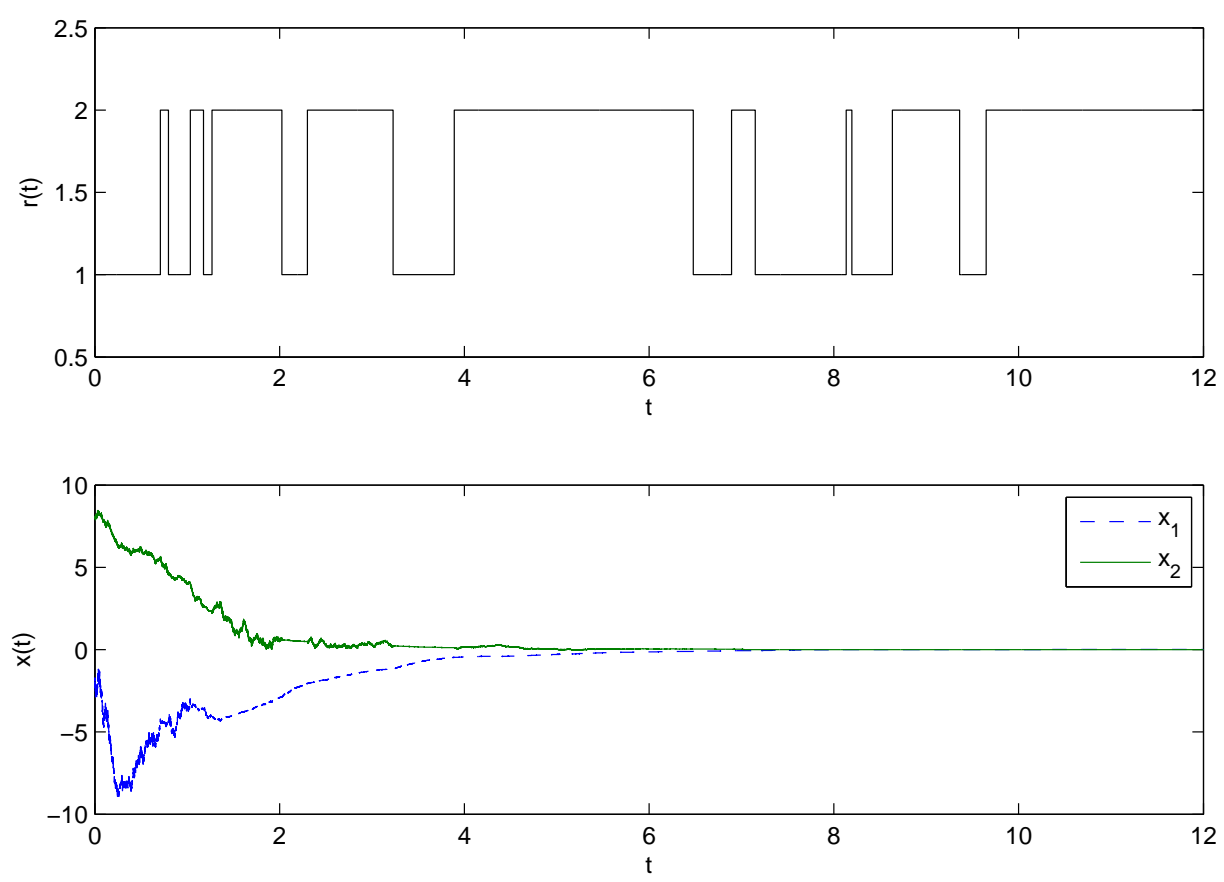

Figure 2: Output injection: the trajectories of the closed-loop system
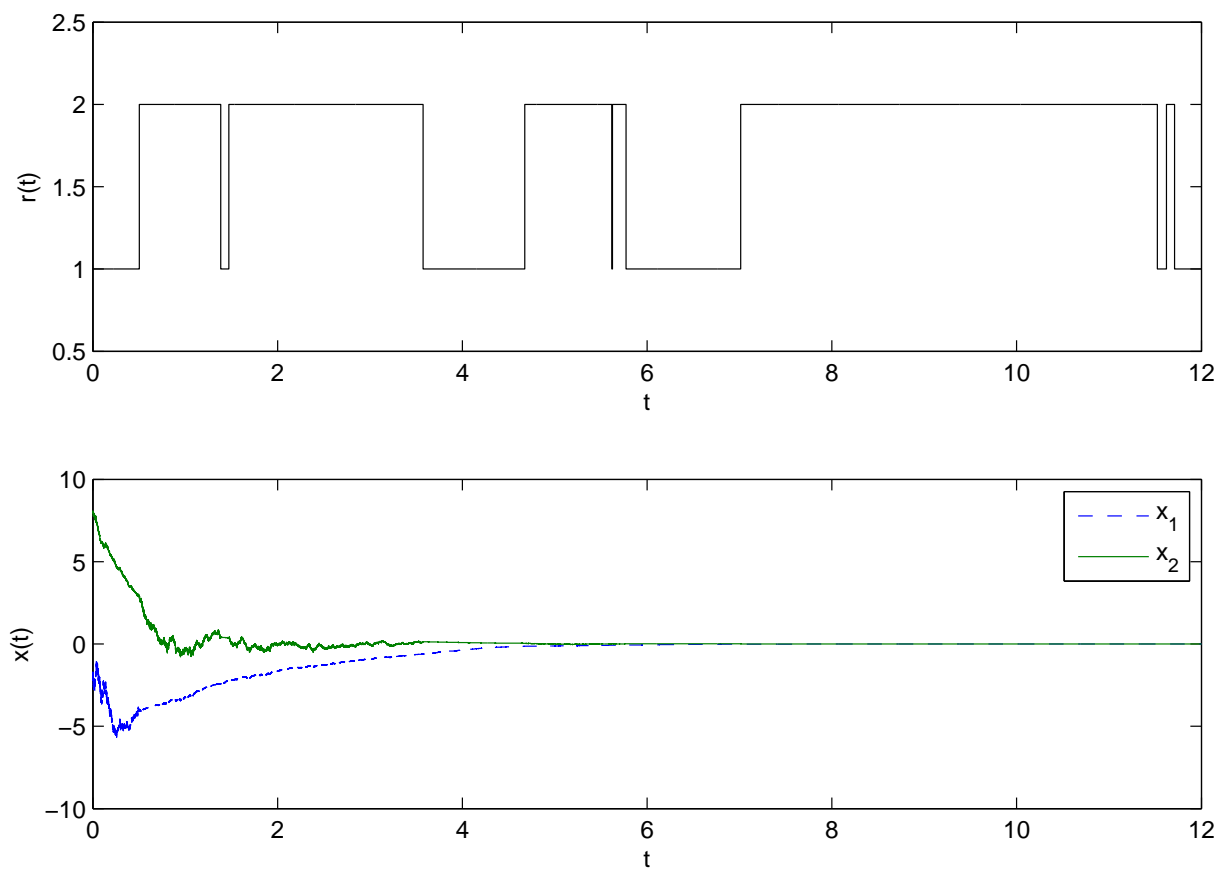

Figure 3: State feedback: the trajectories of the closed-loop system 
Furthermore, $\rho_{1}^{1}, \rho_{2}^{1}, m, \mathcal{L}_{1}^{(1,0)}, \mathcal{L}_{2}^{(1,0)}$ and $\Gamma$ are the same as those presented in Example 1. Our aim is to find $E_{1}$ and $E_{2}$ in $\mathbb{R}^{2 \times 1}$ and then assure $\tau$ is sufficiently small for the controlled system (1) to be exponentially stable in mean square. In what follows, based on Remark 6 , we get a desired feedback controller in the form of (9) with

$$
E_{1}=\left[\begin{array}{l}
-1.4763 \\
-3.3917
\end{array}\right], \quad E_{2}=\left[\begin{array}{l}
-0.8626 \\
-5.9478
\end{array}\right]
$$

A further calculation shows that

$$
\begin{aligned}
\bar{\lambda} & =-48.7227, \quad G_{Q E K}=188.6220, \quad \bar{G}_{Q E K}=101.5372, \quad \zeta=0.0077, \\
M_{K} & =29.2376, \quad \lambda_{M}=69.5636, \quad \tilde{G}_{E}=6.9100, \quad G_{\Lambda K}=0.5492, \quad \tilde{G}_{K}=1.04 .
\end{aligned}
$$

We can see that (33) is satisfied as $\tau^{*}=0.0001303$. To verify the designed controller, Figure 3 shows the trajectories of the closed-loop system for the aforementioned initial conditions. In this case, the nonlinear functions are chosen as $f_{1}(x(t))=f_{2}(x(t))=\left[\begin{array}{c}0.007 \sin \left(e^{-x^{(2)}(t)}\right) \\ 0.0077 \sin \left(e^{-x^{(2)}(t)}\right)+0.0077 \cos \left(x^{(1)}(t)\right)\end{array}\right]$. Moreover, the maximum allowable Lipschitz constant $\zeta^{*}=0.8225$ can be obtained by solving the corresponding optimization problem.

\section{Conclusion}

In this paper, we have shown that unstable hybrid stochastic systems can be stabilized by the quantized feedback controllers based on discrete-time observations of state and mode. Our focus has been on the existence and synthesis of quantized feedback controllers such that the resulting closed-loop systems are mean-square exponentially stable. The significant contribution of this paper is the discrete-time feedback controls designed. The quantization as well as the feedback controls based on discrete-time state and mode observations reduce the burden of communication. Two examples with computer simulations have also been provided to illustrate the effectiveness of the proposed approach. From above-mentioned examples, the bound on $\tau$ obtained in this paper is a little bit conservative. It is useful and challenged to obtain a better bound on $\tau$ by developing some new techniques and methods.

\section{Acknowledgements}

This paper was completed when the first author was visiting the Department of Mathematics and Statistics, University of Strathclyde, whose hospitality is greatly appreciated. This work was supported by the following grants: National Natural Science Foundation of P.R. China (No. 61503190), Natural Science Foundation of Jiangsu Province (No. BK20150927), The Startup Foundation for Introducing Talent of NUIST (No. S8113107001), Natural Science Fundamental Research Project of Jiangsu Colleges and Universities (No. 15KJB120007), Outstanding Youth Science Fund Award of Jiangsu Province (No. BK20140045). The first author would especially like to thank the Chinese Scholarship Council for awarding him the scholarship to visit the University of Strathclyde for one year.

\section{References}

[1] Anderson, W., Continuous-Time Markov Chains, Springer, New York, 1991. 
[2] Mao, X., Yuan, C., Stochastic Differential Equations with Markovian Switching, Imperial College Press: London, 2006.

[3] Mao, X., Stochastic Differential Equations and Their Applications, (Second Edition), Horwood Publishing Limited: Chichester, 2007.

[4] Geromel, J., Gabriel, G., Optimal $H_{2}$ state feedback sampled-data control design of Markov jump linear systems Automatica, 54, 182-188, 2015.

[5] De Souza C., Robust stability and stabilization of uncertain discrete-time Markovian jump linear systems, IEEE Transactions on Automatic Control, 51, 836-841, 2006.

[6] Wang, Z., Liu, Y., Wei, G., Liu, X., A note on control of a class of discrete-time stochastic systems with distributed delays and nonlinear disturbances, Automatica, 46, 543-548, 2010.

[7] Abbaszadeh, M., Marquez, H., LMI optimization approach to robust $H_{\infty}$ observer design and static output feedback stabilization for discrete-time nonlinear uncertain systems, International Journal of Robust and Nonlinear Control, 19, 313-340, 2009.

[8] Shu, H., Yang, H., Wang, Z., Alsaadi, F., Hayat, T., Almost sure $H_{\infty}$ filtering for nonlinear hybrid stochastic systems with mode-dependent interval delays, Journal of the Franklin Institute, 352, 4758$4775,2015$.

[9] Wang, G., Zhang, Q., Yang, C., Robust stabilisation of uncertain delayed Markovian jump systems and its applications, International Journal of Systems Science, 48, 1226-1241, 2017.

[10] Wu, J., Karimi, H., Shi, P., Network-based $H_{\infty}$ output feedback control for uncertain stochastic systems, Information Sciences, 232, 397-410, 2013.

[11] Xu, S., Chen, T., Robust $H_{\infty}$ control for uncertain stochastic systems with state delay, IEEE Transactions on Automatic Control, 47, 2089-2094, 2002.

[12] Mao, X., Lam, J., Huang, L., Stabilisation of hybrid stochastic differential equations by delay feedback control, Systems \& Control Letters, 57, 927-935, 2008.

[13] Mao, X., Stabilization of continuous-time hybrid stochastic differential equations by discrete-time feedback control, Automatica, 49, 3677-3681, 2013.

[14] Mao, X., Liu, W., Hu, L., Luo, Q., Lu, J., Stabilisation of hybrid stochastic differential equations by feedback control based on discrete-time state observations, Systems \& Control Letters, 73, 88-95, 2014.

[15] Mao, X., Almost sure exponential stabilization by discrete-time stochastic feedback control, IEEE Transactions on Automatic Control, 61, 1619-1624, 2016.

[16] You, S., Liu, W., Lu, J., Mao, X., Qiu, Q., Stabilization of hybrid systems by feedback control based on discrete-time state observations, SIAM Journal on Control and Optimization, 53, 905-925, 2015.

[17] Song, G., Zheng, B., Luo, Q., Mao, X., Stabilisation of hybrid stochastic differential equations by feedback control based on discrete-time observations of state and mode, IET Control Theory Applications, 11, 301-307, 2017. 
[18] Fu, M., Xie, L., The sector bound approach to quantized feedback control, IEEE Transactions on Automatic Control, 50, 1698-1711, 2005.

[19] Che, W., Yang, G., Quantised $H_{\infty}$ filter design for discrete-time systems, International Journal of Control, 82, 195-206, 2009.

[20] Zhang, H., Feng, G., Yan, H., Chen, Q., Sampled-data control of nonlinear networked systems with time-delay and quantization, International Journal of Robust and Nonlinear Control, 26, 919-933, 2016.

[21] Tatikonda, S., Sahai, A., Mitter, S., Stochastic linear control over a communication channel, IEEE Transactions on Automatic Control, 49, 1549-1561, 2004.

[22] Wang, Z., Shen, B., Shu, H., Wei, G., Quantized $H_{\infty}$ control for nonlinear stochastic time-delay systems with missing measurements, IEEE Transactions on Automatic Control, 57, 1431-1444, 2012.

[23] Shi, P., Liu, M., Zhang, L., Fault-tolerant sliding-mode-observer synthesis of Markovian jump systems using quantized measurements, IEEE Transactions on Industrial Electronics, 62, 5910-5918, 2015.

[24] Liu, M., Ho, D., Niu, Y., Robust filtering design for stochastic system with mode-dependent output quantization, IEEE Transactions on Signal Processing, 58, 6410-6416, 2010.

[25] Lu, R., Lou, B., Xue, A., Mode-dependent quantised $H_{\infty}$ filtering for Markovian jump singular system, International Journal of Systems Science, 46, 1817-1824, 2015.

[26] Lu, R., Zhao, S., Wu, Y., Xu, Y., Finite-time bounded control for a class of stochastic nonlinear systems with randomly quantized measurements, Journal of the Franklin Institute, 353, 4368-4383, 2016.

[27] Zha, L., Fang, J., Li, X., Liu, J., Event-triggered output feedback $H_{\infty}$ control for networked Markovian jump systems with quantizations, Nonlinear Analysis: Hybrid Systems, 24, 146-158, 2017. 